\title{
Chemical Complementarity in Cometary Dust Particles
}

\author{
LINDSAY P. KELLER ${ }^{1}$ \\ ${ }^{1}$ ARES, Code XI3, NASA Johnson Space Center, USA \\ (lindsay.p.keller@nasa.gov)
}

In carbonaceous chondrites, the major element compositions of chondrules and matrix are chemically complementary suggesting that both formed from a single unfractionated nebular reservoir [e.g. 1]. We have extended this concept to the petrologic components of chondritic porous (CP) interplanetary dust particles (IDPs) that are believed to be derived from comets. While the bulk composition of CP-IDPs are $\sim$ chondritic [2], their petrologic constituents show distinct chemical trends between crystalline and amorphous phases. To detemine if complementarity exists at this scale, we measured the size and composition of all grains within microtome thin sections of CP-IDPs with measured bulk compositions.

We imaged and quantitatively mapped an ultramicrotome thin section from CP-IDP U2015D21 using energy-dispersive $\mathrm{X}$-ray spectrometry in a scanning and transmission electron microscope. From the hyperspectral datasets, we extracted the quantitative major element composition and size (area) of 143 grains in the thin section. The petrography of the IDP is dominated by equilibrated aggregates [3], amorphous silicate grains (GEMS [4]), single crystals of enstatite and forsterite, and opaque grains (FeNi sulfides and $\mathrm{FeNi}$ metal). Amorphous silicate (GEMS) grains are a major component of primitive CP-IDPs and have characteristic element/Si ratios that are $\sim 0.7$ of solar values, one of their fundamental properties [5]. For U2015D21, crystalline material comprises $\sim 68 \%$ of the section area and GEMS grains $\sim 32 \%$. The higher $\mathrm{Mg} / \mathrm{Si}, \mathrm{Al} / \mathrm{Si}, \mathrm{S} / \mathrm{Si}, \mathrm{Ca} / \mathrm{Si}$, and $\mathrm{Fe} / \mathrm{Si}$ area-weighted ratios for crystals combined with the lower ratios in GEMS grains reproduce the bulk composition of U2015D21 as determined by [2].

Systematic measurements of GEMS bulk chemical and isotopic compositions demonstrate that the majority of GEMS grains are of solar system origin [5]. The chemical complementarity between GEMS grains and the crystalline components in CP-IDPs shown here provides further support for a solar system origin. Additional CP-IDPs with variable crystalline/amorphous ratios is underway.

References: [1] Palme, H. et al. (2015) EPSL 411, 11-19. [2] Thomas, K. L. et al. (1993) GCA 57, 1515-1566. [3] Keller, L. P. \& Messenger, S. (2009) LPSC XL, \#2121. [4] Bradley, J. P. (1994) Science 265, 925-929. [5] Keller, L. P. and Messenger, S. (2011) GCA 75, 5336-5365. 